\title{
Bacterial Coating on Seeds as a Potential Application of Bio-inoculants
}

\author{
R Simarmata ${ }^{1}$, Ngadiman ${ }^{2}$, M S Rohman ${ }^{2}$ \\ ${ }^{1}$ Research Center for Biotechnology, Indonesian Institute of Science, Cibinong, Indonesia \\ ${ }^{2}$ Departement of Agricultural Microbiology, Universitas Gadjah Mada, Yogyakarta, Indonesia \\ *Corresponding author. Email: rumella_2001@yahoo.com
}

\begin{abstract}
Seed inoculation with plant growth promoting bacteria (PGPB) is an ideal tool to supply the soil with a high density of beneficial microorganisms. However, viability of microorganisms is a major problem during seed treatment and storage. In this research, seed inoculated by PGPB was conducted to prove the presence and colonization the bacteria on the seed and their impact to the germination and plant growth of soybean. Soybean seed was coated by 4 endophytic bacteria strains (Pseudomonas sp. PIR3C, Pantoea sp. KD6.2, Raoultella sp. PCM8, and Pseudomonas sp. KS12). The ability of the endophytic bacteria to colonize soybean seeds was observed by scanning electron microscopy (SEM). Then, we observe the efficacy of endophytic bacteria inoculation on Soybean growth. The result demonstrated that seed inoculation was able to enter and colonize soybean seed. Seed inoculated with Pseudomonas sp. PIR3C and Raoultella sp. PCM8 significantly increased germination, chlorophyll content, shoot length, root length, and shoot diameter. Furthermore, Pseudomonas sp. PIR3C inoculation increased leaf number and dry weight of the soybean seedlings. Therefore, inoculation method by coating seeds with bacteria could be considered as a promising technique to apply inoculants as biofertilizer in improving plant growth and productivity.
\end{abstract}

Keywords: Bacterial Coating, Bio-inoculants.

\section{INTRODUCTION}

Plants have a complex microbiome of bacteria that able to colonize organs and tissues of plants [1] and cause various effects on the host. Plant growth promoting bacteria (PGPB) are beneficial soil bacteria that colonize plant tissues and cause a positive effect on plants through direct and indirect mechanisms. These functional properties are critical when considering the formulation for biofertilizers, which may be of advantage due to less environmental pollution than chemical applications.

PGPB can stimulate plant growth by producing growth hormones, vitamins and various organic acids, increasing nutrient intake for plants and modulating the amount of plant hormones by activities such as nitrogen fixation, phosphate solubilization, IAA production, cytokinins, and ACCD enzymes. PGPB also play a role as biological control of pathogens, mainly caused by the synthesis of siderophores, antibiosis and induced resistance in crops against a broad spectrum of pests and diseases [2].

Plant growth promoting characteristics can be produced by groups of rhizosphere bacteria and endophytic bacteria. Rhizosphere bacteria are bacteria that grow in the area around plant roots, while endophytic bacteria are bacteria that grow in plant tissues. Endophytic microbes are microscopic life organisms (bacteria and fungi) that live in plant tissues (xylem and phloem), leaves, roots, fruits, and stems. These microbes colonize the internal parts and do not cause infection in host plants. Endophytic microbes live symbiosis mutually beneficial, in this case endophytic microbes get nutrients from the results of plant metabolism and play a role in increasing plant growth, protecting plants against herbivores, insects, disease pests, or pathogenic microbes. Meanwhile, plants get derivatives of nutrients and active compounds needed during their lives [3]. Little information is available about the role of endophytic bacteria as plant growth promoting (PGP). Rashid [4] proved the ability of endophytic bacteria isolated from roots, stems, and leaves of tomato plants as PGP on high-salinity land. The use of endophytic bacteria provides more advantages compared to rhizosphere bacteria with the same activity, especially when bacteria are applied in the field.

Soybean is important crops worldwide, but their production has been severely reduced in recent decades due to soil salinity because this grain legume is highly sensitive to salinity [5]. Thus, a better performance in soybean production based on 
the use of microbial inoculants is needed that increase: rooting ability in the early stages, root surface for nutrient exchange between soil and plants and easy availability of essential nutrients such as nitrogen and phosphorus.

PGPB inoculation constitutes a promising alternative strategy to reduce the use of chemical pesticides and fertilizers. Inoculants can be applied to both seeds and soil. Nevertheless, because land application implies higher volumes of inoculums for suitable distribution of the bacteria, inoculation of seeds is the most effective and economic way of displaying PGPB in the rhizosphere. Thus, seed inoculation with PGPB is an ideal tool to deliver high densities of viable beneficial microorganisms to the soil where emerging plant roots may be colonized by them [6]. However, despite numerous laboratory studies that have demonstrated the ability of beneficial microorganisms to increase plant development, few bioinoculants adhered to seeds are commercially available because maintaining viable microorganisms is a major problem during seed treatment and storage [7].

Inoculation with plant beneficial microorganisms in order to augment the efficacy and quality of bioinoculants and reduce costs and environmental impact. Moreover, these inoculation has been suggested for seed treatment, considering the improved environmental persistence of

\section{MATERIALS AND METHODS}

\subsection{Bacterial strains and soybean seed}

All of the bacterial strains that used in this research are plant growth promoting endophytic bacteria (PGPE) isolated from Special Region of Yogyakarta. The bacteria are Pseudomonas sp. PIR3C, Pseudomonas sp. KS12, Raoultella sp. PCM8, and Pantoea sp. KD6.2. We use Pseudomonas putida UW4 as positive control strain (source: Glick et al.) that previously known as potential PGP bacteria. The bacteria are sub cultured on Nutrient Agar medium and Nutrient Agar Broth. Soybean seed (Glycine max var.Gema) was used in this study.

\subsection{Seed inoculation with bacteria}

The bacterial isolates were grown in Tryptic Soy Agar (TSA) medium at $\pm 28^{\circ} \mathrm{C}$ for $24 \mathrm{~h}$. The inoculants for treating seeds were prepared by suspending cells from agar plates in a TSB medium. Soybean seeds (Glycine max var. Gema) were surface-sterilized with $96 \%$ ethanol for 1 minute followed by immersion in a solution of $4.5 \%$ sodium hypochlorite for 3 minutes and finally microorganisms to improve the effectiveness and consistency of microbial inocula. After this, the bacteria released from the seed must migrate through the soil, facing competitionfrom the native microorganisms. Therefore, a simple and versatile method that directly allows seed coating with encapsulated bioinoculants could be a promising alternative.

Pseudomonas sp. PIR3C, Pseudomonas sp. KS12, Raoultella sp. PCM8, and Pantoea sp. KD6.2 strains were isolated from different ecological niches (paddy, cocoa, paddy and potatoes root tissue, respectively) and selected as plant growth promoting endophytic bacteria (PGPE) candidates for their potential beneficial properties (IAA production, phosphate solubilization, ACCD activity and nitrogen fixation). Soybean seed inoculation with PGPE can protects bacterial cells from adverse environmental conditions, ameliorating their survival during seed storage, improves seed germination, and contributes to root colonization by PGPE, which has a positive impact on plant growth. Thus, the aims of this study have been to evaluate the effect of bacterial inoculation (Pseudomonas sp. PIR3C, Pseudomonas sp. KS12, Raoultella sp. PCM8, and Pantoea sp. KD6.2) in soybean seed on germination and plant growth parameters.

rinses with sterile distilled water ten times. The seeds were dried on a sterile tray for $30 \mathrm{~min}$. The sterilized seeds were treated with Pseudomonas sp. PIR3C, Pseudomonas sp. KS12, Raoultella sp. PCM8, and Pantoea sp. KD6.2, and P. putida UW4 inoculation which were imbibed in bacterial suspension $\left(1 \times 10^{8}\right.$ CFU.seed $\left.^{-1}\right)$ for $2 \mathrm{~h}$, and an excess suspension was drained. Following this, the seeds inoculated with bacteria were stored in sterilized Petri dishes containing a piece of filter paper Whatman no. 40 at $28^{\circ} \mathrm{C}$ under dark conditions. In order to evaluate the bacterial survival of the strains under study, sterile conditions were used during the seed storage time [8].

\subsection{Scanning electron microscopy (SEM)}

Seed soybean was assessed using scanning electron microscopy (SEM). The samples were affixed to sample stubs and subsequently coated with gold. Micrographs were then obtained using JEOL JSM 5310 equipment (Japan) at an acceleration voltage of $10 \mathrm{kV}$ and a sample to detector distance of $5 \mathrm{~mm}$ [9]. 


\subsection{Colonization of PGPE in Inoculated Soybean Seeds}

The presence of endophytic bacteria in soybean seeds inoculated by selected bacteria can be seen by placing the soybean seeds on the Luria Bertani agar media. The treated seeds were soaked with bacterial culture (OD $0.5 ; 10^{7}-10^{8} \mathrm{CFU} / \mathrm{ml}$ ) for 2 hours, then dried on petri which was coated with sterile filter paper. The seeds are then surface-sterilized as in step describe previously, then cut transversely and the cut part is affixed to the media. The same method was done for soybean seeds which were not inoculated with bacteria and acted as a control treatment. Seeds that have been placed on the media are incubated for 7 days in growth chamber, then the growth of bacteria around the soybean seeds placed in the agar medium was observed [8].

\subsection{Germinating Seed Bioassay}

For evaluating the effect of PGPE on the germinating of soybean seeds was accordance with the method described by Zhou et al. [10] with modification. The first, we selected uniformly-sized soybean seeds showing no signs of damage. Sterilized seed was inoculated with bacterial suspension as described above. Seeds both imbibing in medium and were not treated with bacterial inoculation were served as a control (KO) and sown under the same conditions as the inoculated seeds. The bacterized seeds and control were placed in the pre-sterilized petridish containing a piece of filter paper with the addition of $10 \mathrm{ml}$ of sterile deionized water. One hundred pre-germinated seeds for each treatment were used and incubated in the growth chamber at $\pm 28^{\circ} \mathrm{C}$, and $1 \mathrm{ml}$ of sterile distilled water was added to the dishes every $24 \mathrm{~h}$. Over the next 7 days, the germinated seeds were counted. Germination percentage was calculated according to the formula described by Maguire [11]:

Germination percentage $=\frac{\text { number of germinated }}{\text { number of seeds used in th } \mathrm{b}}$

\subsection{Pigment Extraction}

The soybean leaf from inoculated or un-inoculated seed is collected from the same age (35 days after planting). For pigment extraction, $50 \mathrm{mg}$ of the fresh material from the middle part of fully developed leaves of each plant was ground using a pestle and extracted with $0.5 \mathrm{ml} 90 \%$ acetone and $10 \mu \mathrm{M} \mathrm{KOH}$. Mix the extract very well and incubate on the ice for $20 \mathrm{~min}$. The resulting suspension was centrifuged at $4^{\circ} \mathrm{C}$ at $13.000 \mathrm{rpm}$ for $10 \mathrm{~min}$. Chlorophyll and carotenoids contents were determined in acetone supernatants according to the method of Lichtenthaler [12]. The absorbance values were determined with a Shimadzu UV-1601 spectrophotometer at 470; 645; and $663 \mathrm{~nm}$ which was then used to determine total leaf chlorophyll expressed as $\mathrm{mgL}^{-1}[13]$.

\subsection{Growth promotion study}

These studies were performed in the green house of the Faculty of Agronomy of UGM (Universitas Gadjah Mada). Three kilo grams of sterile of sand and husk in a ratio of 3:0.5 were placed in the pots. In each pot, three soybean seeds were seeded at a distance of $1.5 \mathrm{~cm}$ from each other and from the pot edges. Finally, 0.2 gr urea, $0.3 \mathrm{~g}$ SP-36 and $0.2 \mathrm{~g}$ KCL fertilizer were added to each pot with spot placement method, and the pot was reposition every four days. On the 7th day, two out of three soybean seedlings from each cup were removed in order to preserve the one that showed the best growth.The plants were harvested at the end of 35 days to record biometric observations. Leaf number, length of shoot and root and dry biomass were evaluated to determine the effect of treatments on the growth parameters. The shoot and root of the plants were separated and dry weight was determined after drying in an oven at $70^{\circ} \mathrm{C}$ for $48 \mathrm{~h}$.

\section{RESULTS AND DISCUSSION}

\subsection{Colonization of soybean seeds by PGPE}

This experiment was carried out to see the presence and colonization of endophytic bacteria inoculated in soybean seeds. Based on the results of experiments, the bacterial growth was not found around the seeds that were not inoculated with bacteria (control), whereas around bacteria inoculated seeds, the bacterial growth was found on the media (Figure. 1). This shows that endophytic bacteria have the ability to colonize soybeans inoculated with bacteria. The results of this experiment still need to be proven further by observing Scanning Electron Microscopy (SEM) and molecular analysis such as Ribosomal RNA Intergenic spacer Analysis RISA in subsequent studies [14]. 

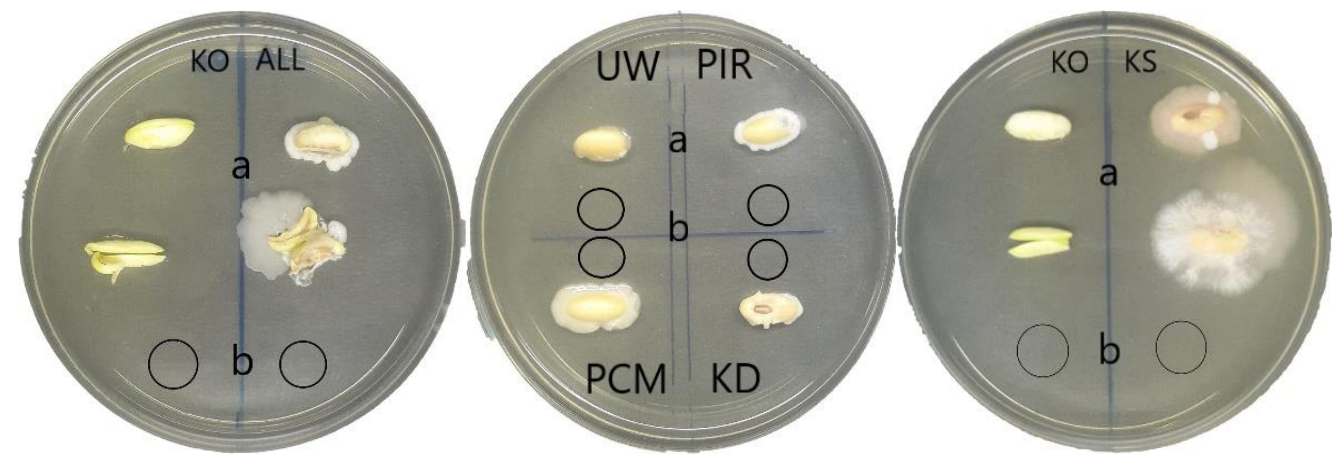

Figure. 1. Soybean Seed Macro-appearance. (a). Sterilized soybean seeds and planted on TSA media, (b). Water aliquot from the final rinse solution. $\mathrm{KO}=$ seed without treatment, ALL $=$ seed with inoculation of 4 potential bacteria (PIR3C, PCM8, KS12, and KD6.2), UW = seed with inoculation of bacterial strain P. putida UW4, PIR $=$ seed with inoculation of bacterial strain Pseudomonas sp. PIR3C, KD = seed with inoculation of Pantoea sp. KD6.2, PCM8 = seed with inoculation of strains of Raoultella sp. PCM8, KS = seed with inoculation of Pseudomonas sp. KS12.

The ability of Pseudomonas sp. PIR3C, Pseudomonas sp. KS12, Raoultella sp. PCM8, and Pantoea sp. KD6.2 to colonize soybean seeds from treated seeds was evaluated. Scanning electron microscopy revealed that soybean seeds from seeds subjected to inoculation with Pseudomonas sp. PIR3C, Pseudomonas sp. KS12, Raoultella sp. PCM8, and Pantoea sp. KD6.2 culture were successfully colonized by all of PGPE (Figure 2B, $2 \mathrm{C}, 2 \mathrm{D}, 2 \mathrm{E})$. Figure 2 shows a visibly higher microorganism colonization on the soybean seeds with seeds inoculated with bacterial cultures compared to seeds untreated. As expected, bacterial presence was not evidenced in the seeds from the untreated seeds (Figure 2A1 and A2).

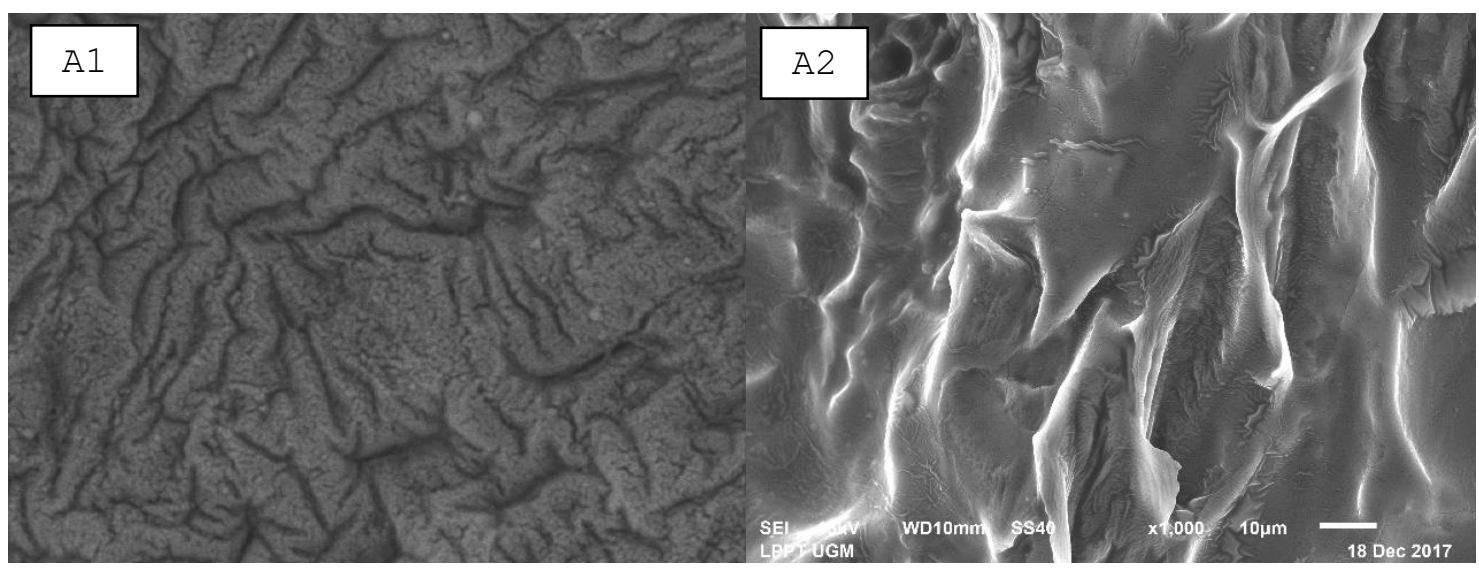



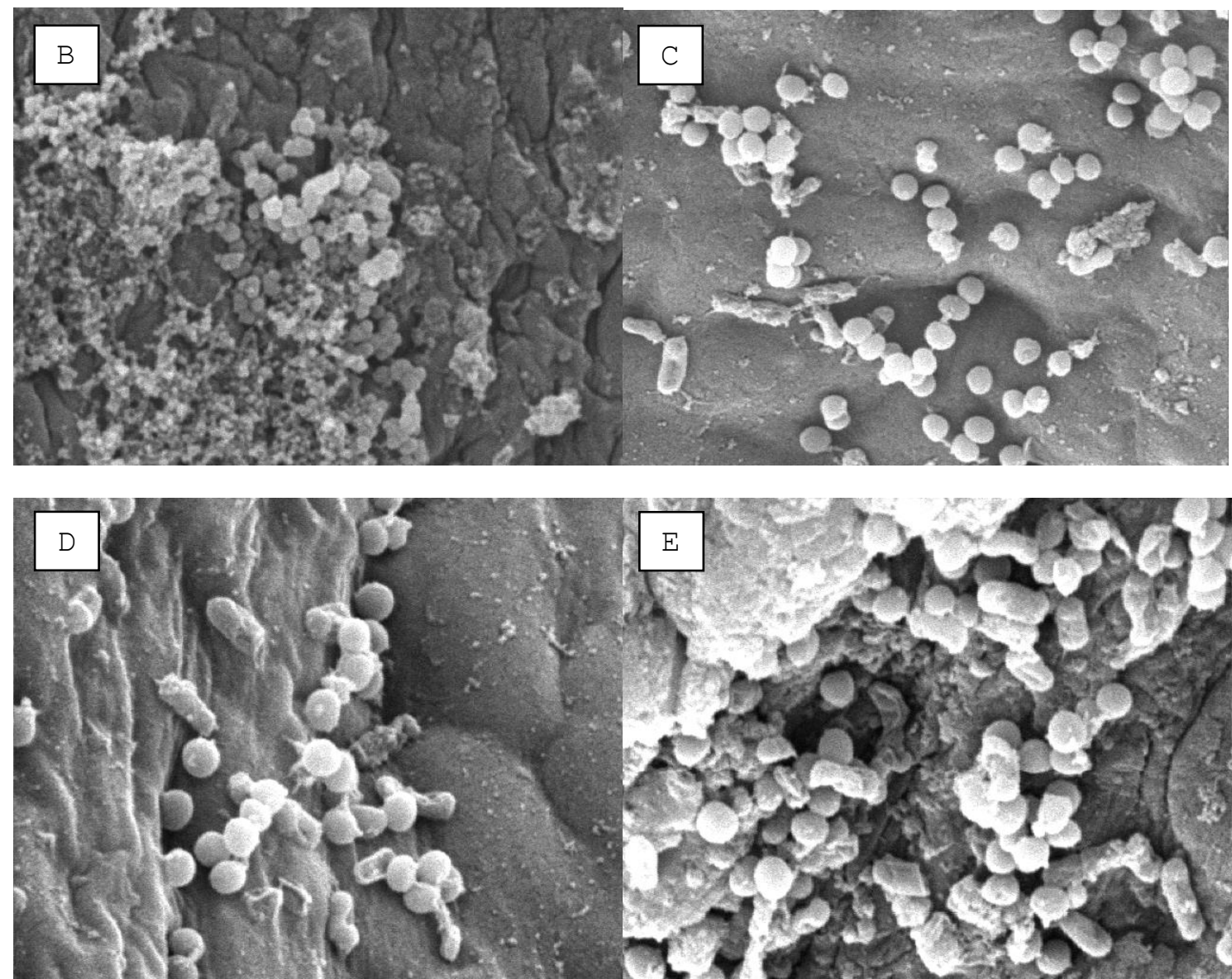

Figure 2. Micro-appearance of soybean seeds (Glycine max var. Gema variety). (A) Scanning electron microscopy micrographs of untreated seeds (Untreated), (B) inoculated with Pseudomonas sp. PIR3C, (C) Pseudomonas sp. KS12, (D) Raoultella sp. PCM8, and (E) Pantoea sp. KD6.2 cultures. The micrographs were observed at 5000x magnification (A1, B, C, D, E), and 1000x magnification (A2).

\subsection{Effect of PGPE inoculation on soybean germination}

Inoculating seeds with strain Pseudomonas sp. PIR3C, Pseudomonas sp. KS12, Raoultella sp. PCM8, and Pantoea sp. KD6.2 showed the improvement of germination compared to the uninoculated seeds. Seeds inoculated with strain
Pseudomonas sp. PIR3C showed the maximum germination percentage, which was $13.34 \%$ higher than un-inoculated control, followed by seeds inoculated with strains Raoultella sp. PCM8 and $P$. putida UW 4. This may be due to the PGP activity of Pseudomonas sp. PIR3C such as ACCD activity, is higher than the other bacteria that inoculated 


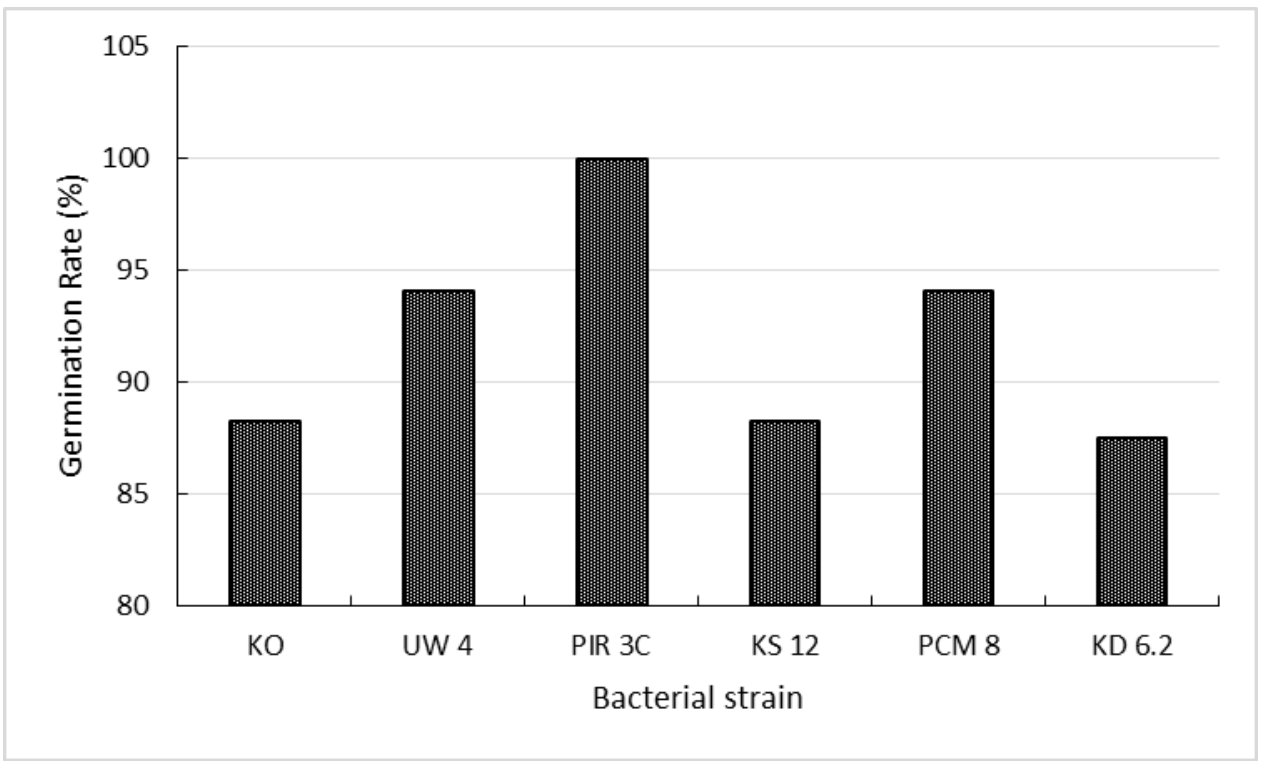

Figure 3. Effect of PGPE inoculation on germination rate of soybean. (KO = un-inoculated bacteria, UW4 = inoculated with $P$. putida UW4, PIR3C = Pseudomonas sp. PIR3C, KS12 = Pseudomonas sp. KS12, PCM8 = Raoultella sp. PCM8, KD6.2 = Pantoea sp. KD6.2).

The inoculation of soybean seeds with PGPE did not only improve the growth of soybean but also enhance seed germination. The enhanced germination may be due to their ability as plant growth promoting by PGP substance of bacteria. This process improved bacterial survival in seeds stored at room temperature for $48 \mathrm{~h}$. further studies are required to evaluate other methodology to improve microorganism survival, seed characterization, and plant growth after the process. A possible reason for the above is that the bacterial insertion was capable of limiting access to toxic substances and controlling environmental factors. However, inhibition of in vitro soybean germination by PGPE ( $P$ Pseudomonas sp. KS12 and Pantoea sp. KD6.2), probably due to nutrient competition between seeds and bacteria, has also been reported [15].

\subsection{Growth promotion in soybean}

The characteristic of plant growth promotion from bacterial inoculation demonstrated with significantly increasing of indicator parameter bacterial-treated seeds compared to the un-treated seeds. The plants from the bacterial inoculation- treated seeds showed a higher chlorophyll content than those from the un-treated seeds (Figure 4A). Moreover, inoculation with Pseudomonas sp. PIR3C increasing leaf number in comparison with the controls (Figure 4B). On the other hand, root length was not affected significantly by any of the treatments (Figure 4C), whereas an increase in shoot length was induced with the inoculation of the seeds with Pseudomonas sp. PIR3C, Pseudomonas sp. KS12, Raoultella sp. PCM8, and Pantoea sp. KD6.2, respectively (Figure 4D). The dry weight of the plants from the bacterial-treated seeds was significantly higher compared to the plants from all the treatments, with the exception of those fromthe Pantoea sp. KD6.2-treated seeds (Figure 4E). Moreover, similar results were evidenced in the plants from the Gema variety seeds inoculated with both Pseudomonas sp. PIR3C, Pseudomonas sp. KS12 (Figure 4F). The obtained results in this stage indicate that the beneficial effect Raoultella sp. PCM8, Pseudomonas sp. PIR3C, Pseudomonas sp. KS12, and Pantoea sp. KD6.2 on the different growth parameters was increased with the bacterial inoculation. 

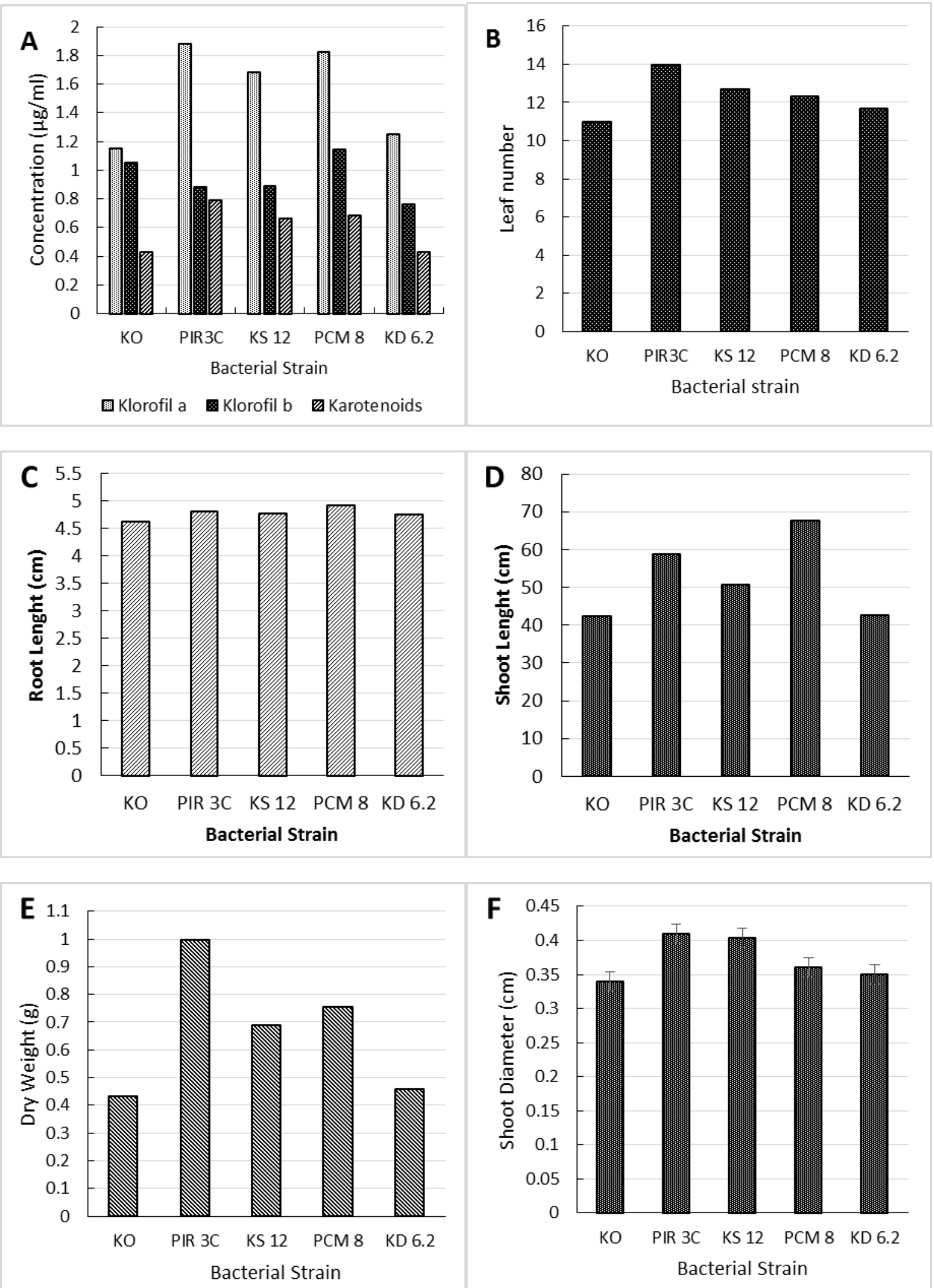

Figure 4. Effect of seed inoculating with endophytic bacteria on growth promotion in soybean plants. (A) pigment content, (B) Leaf number, (C) root length, (D) shoot length, (E) dry weight of shoot and (F) shoot 
diameter determined in plants from seeds (GEMA varieties) inoculated with Raoultella sp. PC 8, Pseudomonas sp. PIR3C, Pseudomonas sp. KS12, and Pantoea sp. KD6.2.

Seed inoculation with endophytic bacteria contributed to bacterial colonization in the roots of soybean, which positively impacted plant growth. It has been reported that native isolates are more suited to the local environment, resulting in competitive advantages over non-indigenous strain [16]. But these results indicate that all isolates used in this study can adapt better to exert its beneficial effects even though the isolates are not a soybean isolate.

\section{CONCLUSION}

Inoculating soybean seeds with Raoultella sp. PCM8, Pseudomonas sp. PIR3C, Pseudomonas sp. KS12, and Pantoea sp. KD6.2 did not affect either the viability of these endophytic bacteria. Seed inoculating with bacteria Raoultella sp. PCM8, Pseudomonas sp. PIR3C, Pseudomonas sp. KS12, and Pantoea sp. KD6.2 improved survival on the seeds and contributed to the successful colonization of all bacteria on the plant seed. Moreover, seed inoculating with Pseudomonas sp. PIR3C increased germination, pigment content, leaf number, shoot diameter and dry weight of the shoot. On the other hand, seed inoculating with Raoultella sp. PCM8 enhanced shoot and root length. Therefore, the isolate applied in the present work to inoculated seeds could be considered a promising eco-friendly biofertilizer approach to improve soybean production through the use of a microbial inoculant. Inoculating seed with bacteria can protects bacteria and seeds from the abiotic stress of the environment and promotes successful inoculant colonization.

\section{ACKNOWLEDGMENTS}

This work was partially funded by the Higher Education of Priority Research Contract No. 015 / SP2H / LT / DRPM /II/2016.

\section{REFERENCES}

[1] Hallman J, Quadt-Hallman A, Mahafee WF, Kloepper JW 1997 Can. J. Microbiol. 43: 895-914.

[2] Fischer H M 1994 Microbiol. Rev. 58 (3): 352-386.

[3] Strobel G, Daisy B 2003 Microbiology and Molecular Biology Reviews 67(4): 491-502.

[4] Rashid S, Charles T C, Glick B R 2012 Applied Soil Ecology 61: 217-224.

[5] Shereen A, Ansari R, Soomro A Q 2001 Pakistan Journal of Botany 33(4): 393-402.

[6] OÂ Callaghan M 2016 Appl Microbiol Biotechnol 100: 5729-5746.
[7] OÂ Callaghan M, Wright D, Swaminathan J, Young S, Wessman P 2012 Agron New Zeal. 42: 149-154.

[8] Govindasamy V, Senthikumar M, Mageshwaran, Annapurna K 2009 J Plant Biochem and Biotech 18(1):71-76.

[9] GoldsteinJ I, Newbury, Echlin, Joy A D, Romig Jr, Lyman, Fiori, Lifshin E $19922^{\text {nd }}$ ed. Plemun Press, New York, 820p.

[10] Zhou G C, Wang Y, Zhai S, Ge F, Liu Z H, Dai Y J 2013 Appl Microbiol Biotechnol. 97: 4065-4074.

[11] Maguire J.D 1962 Crop Sci 2:176-177.

[12] Lichtenthaler N K 1987 Methods enzymology 148: 350-382.

[13] El Sabagh A, Omar A E, Saneoka H, Barutcular C 2015 J. Agr. Sci 25(3): 269-284.

[14] Sigler WV, Crivii S, Zeyer J 2002 Microb. Ecol. 44 (4): 306-316.

[15] Ştefan M, Mihăşan M, Dunca S 2008 Secţiunea Genet ŞiBiol Mol. IX: 105-110.

[16] Santoro MV, Cappellari L R, Giordano W, Banchio E 2015 Plant Biol. 17: 1218-1226. 